\title{
Inverse problem for cracked inhomogeneous Kirchhoff-Love plate with two hinged rigid inclusions
}

Nyurgun Lazarev ${ }^{1 *}$ (1)

"Correspondence: nyurgun@ngs.ru 'North-Eastern Federal University, Belinsky str., 58, 677000 Yakutsk,

Russian Federation

\section{Springer}

\begin{abstract}
We consider a family of variational problems on the equilibrium of a composite Kirchhoff-Love plate containing two flat rectilinear rigid inclusions, which are connected in a hinged manner. It is assumed that both inclusions are delaminated from an elastic matrix, thus forming an interfacial crack between the inclusions and the surrounding elastic media. Displacement boundary conditions of an inequality type are set on the crack faces that ensure a mutual nonpenetration of opposite crack faces. The problems of the family depend on a parameter specifying the coordinate of a connection point of the inclusions. For the considered family of problems, we formulate a new inverse problem of finding unknown coordinates of a hinge joint point. The continuity of solutions of the problems on this parameter is proved. The solvability of this inverse problem has been established. Using a passage to the limit, a qualitative connection between the problems for plates with flat and bulk hinged inclusions is shown.
\end{abstract}

MSC: $49 \mathrm{~N} 45 ; 49 J 40$

Keywords: Variational inequality; Inverse problem; Nonpenetration; Nonlinear boundary conditions; Crack; Rigid inclusion

\section{Introduction}

The success of the modern industry is largely based on the widespread use of composite materials. Mathematical modeling of the properties of composites, their behavior under certain conditions provide opportunities for optimizing the parameters and characteristics of composites. As a result, qualitatively new models as well as various complex mathematical approaches leading to new formulations of problems are being developed. Along with the advantages of composite materials, the difference in the physical characteristics of the components can lead to cracks and, as a result, to partial damages to structural elements or destructions of entire composite structures. In some cases, in practice, it is required to assess the possible consequences due to partial damage to some structural elements. Since it is not always possible to find out damage characteristics of hidden structural elements directly, it is necessary to look for methods to determine them indirectly.

(c) The Author(s) 2021. This article is licensed under a Creative Commons Attribution 4.0 International License, which permits use sharing, adaptation, distribution and reproduction in any medium or format, as long as you give appropriate credit to the original author(s) and the source, provide a link to the Creative Commons licence, and indicate if changes were made. The images or other third party material in this article are included in the article's Creative Commons licence, unless indicated otherwise in a credit line to the material. If material is not included in the article's Creative Commons licence and your intended use is not permitted by statutory regulation or exceeds the permitted use, you will need to obtain permission directly from the copyright holder. To view a copy of this licence, visit http://creativecommons.org/licenses/by/4.0/. 
For a loaded body, the presence of an inclusion or a crack means that there can be significant stress values in the vicinity of these inhomogeneities. For this reason, the choice of one or another method for specifying the boundary conditions characterizing mechanical processes near a crack can affect the physical adequacy of an entire mathematical model as a whole.

In this work, we follow the nonlinear modeling that uses Signorini-type boundary conditions on the crack faces which was developed in [1-13] and other works. These nonlinear conditions need to use variational formulations. Within the variational approach, equilibrium problems for composite bodies with elastic or rigid inclusions have been successfully formulated and investigated, see for example [14-28]. The other challenge of our work concerns the variation of inclusion shapes. The shape and topological sensitivity analysis of related variational problems was developed in [29-35] and other papers. In particular, for a crack problem subject to nonpenetration, an optimal control problem concerning minimization of the Griffith functional with respect to the shape and the location of a small elastic inclusion was investigated in [36,37]. Explicit formulae of derivatives of the energy functionals with respect to the shape variation of rigid inclusions were obtained in $[19,24]$. Inverse problems for the nondestructive determination of elastic and rigid inclusions embedded in Kirchhoff-Love bending elastic plates were investigated in [38-40]. We refer the reader to convergence results for elliptic variational-hemivariational inequalities [12] and for double phase obstacle problems with multivalued convection term [13].

Currently we are dealing with a nonlinear model describing a plate with two flat rigid inclusions which are connected in a hinged manner. Namely, we suppose that both inclusions have a common hinge fiber. In addition to this, we assume that both inclusions are delaminated from an elastic media, thus forming an interfacial crack. A new inverse problem predicting the structural integrity of a composite plate is formulated. It is assumed that we know that the plate has two hinged inclusions, but simultaneously we do not know the location of a hinge fiber. Under the assumption that the sum of lengths of the inclusions is constant, a solvability of this inverse problem is proved.

\section{Family of equilibrium problems}

Let $\Omega \subset \mathbb{R}^{2}$ be a bounded domain with a boundary $\Gamma$ of class $C^{1,1}$. Suppose that a rectilinear curve $\gamma=(-1,1) \times\{0\}$ lies strictly inside $\Omega$, i.e., $\bar{\gamma} \subset \Omega$. We assume that $\Gamma$ consists of two disjoint curves $\Gamma=\Gamma_{0} \cup \Gamma_{1}$, where meas $\left(\Gamma_{0}\right)>0$ and meas $\left(\Gamma_{1}\right)>0$. We suppose that the domain $\Omega$ can be split into two subdomains $\Omega_{1}$ and $\Omega_{2}$ with Lipschitz boundaries $\partial \Omega_{1}$ and $\partial \Omega_{2}$ such that $\gamma \subset \partial \Omega_{1} \cap \partial \Omega_{2}$, and meas $\left(\partial \Omega_{i} \cap \Gamma_{0}\right)>0, i=1$, 2. The latter condition is important for Korn's inequality to hold in the non-Lipschitz domain $\Omega_{\gamma}=\Omega \backslash \bar{\gamma}$. For simplicity, the plate is assumed to have a uniform thickness $2 h=2$. Let us assign a threedimensional Cartesian space $\left\{x_{1}, x_{2}, z\right\}$ with the set $\left\{\Omega_{\gamma}\right\} \times\{0\} \subset \mathbf{R}^{3}$ corresponding to the middle plane of the plate. The curve $\gamma$ defines a crack (a cut) in the plate. This means that the cylindrical surface of the through crack may be defined by the relations $x=\left(x_{1}, x_{2}\right) \in \gamma$, $-1 \leq z \leq 1$, where $|z|$ is the distance to the middle plane. Depending on the direction of the normal $v=(0,1)$ to $\gamma$, we will keep in mind the positive face $\gamma^{+}$and the negative face $\gamma^{-}$of the curve $\gamma$. The jump [q] of an arbitrary function $q$ across the curve $\gamma$ is found by the formula $[q]=\left.q\right|_{\gamma^{+}}-\left.q\right|_{\gamma^{-}}$. We assume that the plate has two hinged flat inclusions at the positive face of the curve $\gamma$. For a fixed parameter $\delta \in\left[-\frac{1}{2}, \frac{1}{2}\right]$, the curve $\gamma$ is split into two rectilinear curves $\gamma_{1}^{\delta}=(-1, \delta) \times\{0\}$ and $\gamma_{2}^{\delta}=(\delta, 1) \times\{0\}$ corresponding to two flat rigid inclusions (see Fig. 1). 
Figure 1 Objects on the middle plane of the plate

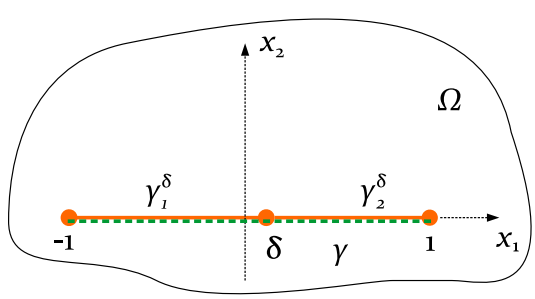

Thus, for the initial undeformed state of the plate, the flat rigid inclusions are specified by the sets $\gamma_{i}^{\delta} \times[-1,1], i=1,2$, and a fiber of a hinge joint is given by the line segment $\{\delta\} \times\{0\} \times[-1,1]$. The elastic part of the plate corresponds to the domain $\Omega_{\gamma}$.

Denote by $\chi=\chi(x)=(W, w)$ the displacement vector of the mid-surface points $\left(x \in \Omega_{\gamma}\right)$, by $W=\left(w_{1}, w_{2}\right)$ the displacements in the plane $\left\{x_{1}, x_{2}\right\}$, and by $w$ the displacements along the axis $z$. The strain and integrated stress tensors are denoted by $\varepsilon_{i j}=\varepsilon_{i j}(W), \sigma_{i j}=\sigma_{i j}(W)$, respectively [1]:

$$
\varepsilon_{i j}(W)=\frac{1}{2}\left(\frac{\partial w_{j}}{\partial x_{i}}+\frac{\partial w_{i}}{\partial x_{j}}\right), \quad \sigma_{i j}(W)=a_{i j k l} \varepsilon_{k l}(W), \quad i, j=1,2
$$

where $\left\{a_{i j k l}\right\}$ is the given elasticity tensor, assumed to be symmetric and positive definite

$$
\begin{aligned}
& a_{i j k l}=a_{k l i j}=a_{j i k l}, \quad i, j, k, l=1,2, a_{i j k l} \in L^{\infty}\left(\Omega_{\gamma}\right), \\
& a_{i j k l} \xi_{i j} \xi_{k l} \geq c_{0}|\xi|^{2}, \quad \forall \xi, \xi_{i j}=\xi_{j i}, i, j=1,2, c_{0}=\mathrm{const}>0 .
\end{aligned}
$$

A summation convention over repeated indices is used in the sequel. Next we denote the bending moments by the formulas [1]

$$
m_{i j}(w)=d_{i j k l} w, k l, \quad i, j=1,2\left(w, k l=\frac{\partial^{2} w}{\partial x_{k} \partial x_{l}}\right)
$$

where the tensor $\left\{d_{i j k l}\right\}$ has the same properties as the tensor $\left\{a_{i j k l}\right\}$. Let $B(O, \cdot, \cdot)$ be a bilinear form defined by the equality

$$
B(O, \chi, \bar{\chi})=\int_{O}\left\{\sigma_{i j}(W) \varepsilon_{i j}(\bar{W})+m_{i j}(w) \bar{w}_{i j}\right\} d x,
$$

where $\chi=(W, w), \bar{\chi}=(\bar{W}, \bar{w})$, and $O$ is some measurable subset of $\Omega$. The potential energy functional of the plate has the following representation [1]:

$$
\Pi(\chi)=\frac{1}{2} B\left(\Omega_{\gamma}, \chi, \chi\right)-\int_{\Omega_{\gamma}} F \chi d x, \quad \chi=(W, w)
$$

where vector $F=\left(f_{1}, f_{2}, f_{3}\right) \in L_{2}(\Omega)^{3}$ describes given body forces [1]. Introduce the Sobolev spaces

$$
\begin{aligned}
& H_{\Gamma_{0}}^{1}\left(\Omega_{\gamma}\right)=\left\{v \in H^{1}\left(\Omega_{\gamma}\right) \mid v=0 \text { on } \Gamma_{0}\right\}, \\
& H_{\Gamma_{0}}^{2}\left(\Omega_{\gamma}\right)=\left\{v \in H^{2}\left(\Omega_{\gamma}\right) \mid v=\frac{\partial v}{\partial n}=0 \text { on } \Gamma_{0}\right\},
\end{aligned}
$$




$$
H\left(\Omega_{\gamma}\right)=H_{\Gamma_{0}}^{1}\left(\Omega_{\gamma}\right)^{2} \times H_{\Gamma_{0}}^{2}\left(\Omega_{\gamma}\right)
$$

where $n=\left(n_{1}, n_{2}\right)$ is the normal vector to $\Gamma$. Note that the following inequality

$$
B\left(\Omega_{\gamma}, \chi, \chi\right) \geq c\|\chi\|^{2} \quad \forall \chi \in H\left(\Omega_{\gamma}\right)\left(\|\chi\|=\|\chi\|_{H\left(\Omega_{\gamma}\right)}\right)
$$

with a constant $c>0$ independent of $\chi$ holds for the bilinear form $B\left(\Omega_{\gamma}, \cdot, \cdot\right)[1]$.

Remark 1 Inequality (1) yields the equivalence of the standard norm and the semi-norm determined by the bilinear form $B\left(\Omega_{\gamma}, \cdot, \cdot\right)$ in the space $H\left(\Omega_{\gamma}\right)$.

The condition of mutual nonpenetration of opposite faces of the crack is given by $[1,16]$

$$
[W v] \geq\left|\left[\frac{\partial w}{\partial v}\right]\right| \quad \text { on } \gamma\left(W v=w_{i} v_{i}\right)
$$

Due to presence of flat rigid inclusions in the plate, restrictions of the functions describing displacements $\chi$ to the corresponding curves $\gamma_{1}^{\delta}, \gamma_{2}^{\delta}$ satisfy a special kind of relations. We introduce the following space which allows us to characterize the properties of the flat rigid inclusions:

$$
R(S)=\left\{\zeta \mid \zeta(x)=\left(b^{i} x_{2}+c_{1}^{i},-b^{i} x_{1}+c_{2}^{i}, a_{0}^{i}+a_{1}^{i} x_{1}+a_{2}^{i} x_{2}\right) ; x \in S\right\}
$$

where $b^{i}, c_{1}^{i}, c_{2}^{i}, a_{0}^{i}, a_{1}^{i}, a_{2}^{i} \in \mathbb{R}, i=1,2$, and $S$ is some subset of $\Omega$ (see $\left.[15,16]\right)$. Suppose that displacements on two flat rigid inclusions have the following properties [11]:

$$
\begin{aligned}
& \left.\chi\right|_{\gamma_{i}^{\delta+}}=\zeta^{i}, \\
& \frac{\partial w}{\partial x_{1}}=-a_{1}^{i}, \quad \frac{\partial w}{\partial x_{2}}=-a_{2}^{i} \quad \text { on } \gamma_{i}^{\delta+}
\end{aligned}
$$

where $\chi=(W, w), \zeta^{i} \in R\left(\bar{\gamma}_{i}^{\delta}\right), i=1,2$. Since there is the hinge joint of inclusions, we assume that the displacements and angles of rotation of the normal fibers of both inclusions are equal at the corresponding point $x^{\delta}=(\delta, 0)$ of the middle plane of the plate

$$
\begin{aligned}
& \zeta^{1}\left(x^{\delta}\right)=\zeta^{2}\left(x^{\delta}\right), \quad \text { where }\left.\chi\right|_{\gamma_{i}^{\delta+}}=\zeta^{i}, \zeta^{i} \in R\left(\bar{\gamma}_{i}^{\delta}\right), i=1,2, \\
& \frac{\partial w}{\partial x_{1}}=-a_{1}^{1}=-a_{1}^{2}, \quad \frac{\partial w}{\partial x_{2}}=-a_{2}^{1}=-a_{2}^{2} .
\end{aligned}
$$

The variational formulation describing the equilibrium state for the elastic plate with the flat delaminated rigid inclusions can be formulated as follows:

$$
\Pi\left(\xi^{\delta}\right)=\inf _{\eta \in K^{\delta}} \Pi(\eta)
$$

For fixed $\delta \in[-1 / 2,1 / 2]$, let $K^{\delta}$ denote the set of admissible displacements

$$
K^{\delta}=\left\{\chi \in H\left(\Omega_{\gamma}\right) \mid \chi \text { satisfies (2), (3), (4) }\right\} .
$$


Remark 2 It should be noted that the conditions $\chi \in H\left(\Omega_{\gamma}\right)$ and (3), (4) imply that equalities (5), (6) hold.

Remark 3 We should note that the notions of flat inclusion and thin inclusions (see $[15,16])$ in the same way are specified with the help of cylindrical surfaces, but for flat inclusions there are additional relations describing a connection of plate deflections $w$ and angles of rotation of the normal fibers $\frac{\partial w}{\partial x_{i}}, i=1,2$, of an inclusion, see (4). Since bulk (volume) rigid inclusions in Kirchhoff-Love plates are defined with the help of sets of the following type $\mathcal{O} \times[-1,1]$, where $\mathcal{O}$ is some subdomain lying in the middle plane of the plate [14]; then these relationships (4) for partial derivatives are naturally fulfilled for bulk (volume) rigid inclusions in $\mathcal{O}$.

One can check by simple reasonings that the set $K^{\delta}$ is convex and closed. The coercivity and weak semicontinuity of the energy functional together with (1) ensure that problem (7) has a unique solution. Moreover, by the Gateaux differentiability of $\Pi$, problem (7) is equivalent to the following variational inequality (see [1]):

$$
\xi^{\delta} \in K^{\delta}, \quad B\left(\Omega_{\gamma}, \xi^{\delta}, \chi-\xi^{\delta}\right) \geq \int_{\Omega_{\gamma}} F\left(\chi-\xi^{\delta}\right) d x \quad \forall \chi \in K^{\delta} .
$$

\section{Inverse problem for a hinge fiber}

In this section we formulate a new inverse problem for the composite plate containing two flat rigid inclusions. This problem is motivated by prediction of the structural integrity of composite bodies. In order to formulate the inverse problem, we suppose that the parameter $\delta$ is assumed to be unknown. Let us introduce the cost functional describing the deviation from some given observation $\chi_{g} \in L^{2}\left(\Gamma_{1}\right)^{3}$

$$
J\left(\xi^{\delta}\right)=\left\|\xi^{\delta}-\chi_{g}\right\|_{L^{2}\left(\Gamma_{1}\right)^{3}}^{2}
$$

More generally, we consider an arbitrary weakly continuous functional of cost $J(\chi)$ : $H\left(\Omega_{\gamma}\right) \mapsto \mathbb{R}$. As commonly adopted in optimal control theory, we should either minimize: find $p_{1} \in R$ such that

$$
p_{1}=\min _{\delta \in[-1 / 2,1 / 2]} J\left(\xi^{\delta}\right),
$$

or maximize the cost: find $p_{2} \in R$ such that

$$
p_{2}=\max _{\delta \in[-1 / 2,1 / 2]} J\left(\xi^{\delta}\right) .
$$

Then, for given intermediate $p \in\left[p_{1}, p_{2}\right]$, we can formulate the inverse problem as follows. Find a parameter $\delta \in[-1 / 2,1 / 2]$ and a displacement field $\xi^{\delta}$ of the body defined in $\Omega_{\gamma}$ such that

$$
\begin{aligned}
& \xi^{\delta} \in K^{\delta}, \quad B\left(\Omega_{\gamma}, \xi^{\delta}, \chi-\xi^{\delta}\right) \geq \int_{\Omega_{\gamma}} F\left(\chi-\xi^{\delta}\right) d x \quad \forall \chi \in K^{\delta}, \\
& p=J\left(\xi^{\delta}\right) .
\end{aligned}
$$

We prove a solution existence of problem (11), (12) fitting suitable values of $p$. The following statement takes place. 
Theorem 1 There exist finite numbers $p_{1}$ and $p_{2}$ solving minimization (9) and maximization (10) problems, with $p_{1} \leq p_{2}$, such that for any fixed $p \in\left[p_{1}, p_{2}\right]$ the inverse problem (11), (12) has a solution.

Proof Let us introduce the function $h:[-1 / 2,1 / 2] \mapsto \mathbb{R}$

$$
h(\delta)=J\left(\xi^{\delta}\right)
$$

where $\xi^{\delta}$ is the solution of the forward problem (8). Our first step is to prove that the function $h$ is continuous on the set $[-1 / 2,1 / 2]$. For fixed $\delta \in[-1 / 2,1 / 2]$, we can substitute test functions $\chi=\xi^{\delta}, \chi=0$ into (8). This gives

$$
\xi^{\delta} \in K^{\delta}, \quad B\left(\Omega_{\gamma}, \xi^{\delta}, \xi^{\delta}\right)=\int_{\Omega_{\gamma}} F \xi^{\delta} d x
$$

and as a consequence the uniform boundedness

$$
\left\|\xi^{\delta}\right\| \leq C
$$

where $C>0$ does not depend on $\delta$. If we prove that for every fixed sequence $\left\{\delta_{n}\right\} \subset$ $[-1 / 2,1 / 2]$ converging to some $\delta \in[-1 / 2,1 / 2]$ there exists a subsequence $\left\{\delta_{k}\right\} \subset\left\{\delta_{n}\right\}$ such that $\xi^{\delta_{k}} \rightarrow \xi^{\delta}$ strongly in $H\left(\Omega_{\gamma}\right)$, thus we establish the strong convergence $\xi^{\delta_{n}} \rightarrow \xi^{\delta}$ in $H\left(\Omega_{\gamma}\right)$ for an arbitrary sequence $\left\{\delta_{n}\right\}$ converging to $\delta$. Let an arbitrary sequence $\left\{\delta_{n}\right\} \subset$ $[-1 / 2,1 / 2]$ be given with $\delta_{n} \rightarrow \delta, \delta \in[-1 / 2,1 / 2]$. In view of (13) we can extract a subsequence $\left\{\delta_{k}\right\} \subset\left\{\delta_{n}\right\}$ such that

$$
\xi^{\delta_{k}} \rightarrow \tilde{\xi} \quad \text { weakly in } H\left(\Omega_{\gamma}\right) \text { as } k \rightarrow \infty
$$

As the next step we reveal a suitable relationship between the sets of admissible displacements. Namely, we show that for the agreed value $\delta \in[-1 / 2,1 / 2]$, the sequence $\left\{\delta_{k}\right\} \subset[-1 / 2,1 / 2]$, and an arbitrary test function $\chi \in K^{\delta}$, there exists a sequence $\left\{\chi_{k}\right\}$ of functions $\chi_{k} \in K^{\delta_{k}}$ converging strongly in $H\left(\Omega_{\gamma}\right)$ to $\chi$ as $k \rightarrow \infty$. Since $x_{2}=0$ on $\gamma_{i}^{\delta}$, $i=1,2$, the following relations hold for $\chi=(W, w)$ :

$$
\begin{aligned}
& \left.\chi(x)\right|_{\gamma_{i}^{\delta+}}=\zeta^{i}(x)=\left(c_{1}^{i},-b^{i} x_{1}+c_{2}^{i}, a_{0}^{i}+a_{1}^{i} x_{1}\right), \quad x=\left(x_{1}, 0\right) \in \gamma_{i}^{\delta}, i=1,2, \\
& \frac{\partial w}{\partial x_{1}}=-a_{1}^{1}=-a_{1}^{2}, \quad \frac{\partial w}{\partial x_{2}}=-a_{2}^{1}=-a_{2}^{2} \quad \text { on } \gamma_{i}^{\delta+}, i=1,2, \\
& a_{0}^{1}=a_{0}^{2}, \quad c_{1}^{1}=c_{1}^{2}, \quad-b^{1} \delta+c_{2}^{1}=-b^{2} \delta+c_{2}^{2}, \\
& {[W] v \geq\left|\left[\frac{\partial w}{\partial v}\right]\right| \quad \text { on } \gamma,}
\end{aligned}
$$

where the former equation in (15) describes rigid properties of flat inclusions, and (16), (17) reflect the hinged joint at the point $(\delta, 0)$.

Next, we construct a sequence $\left\{\chi_{k}\right\}$ of appropriate vector functions $\chi_{k}=\left(W_{k}, w_{k}\right)$ with $W_{k}=\left(w_{1 k}, w_{2 k}\right), k=1,2, \ldots$ We define its two components in the following simplest way:

$$
w_{k} \equiv w, \quad w_{1 k} \equiv w_{1}, \quad k \in \mathbb{N}
$$


Figure 2 Example of rigid displacements $w_{2}$ along hinged flat inclusions

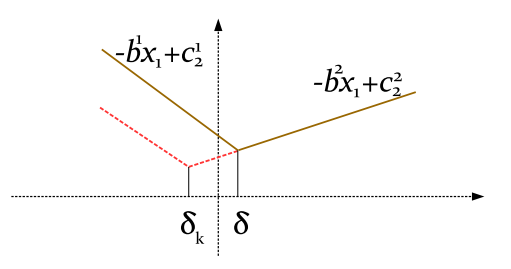

then we have

$$
\begin{aligned}
& \left.w_{k}\right|_{\gamma_{i}^{\delta_{k^{+}}}}=a_{0}^{1}+a_{1}^{1} x_{1}, \quad \frac{\partial w_{k}}{\partial x_{1}}=-a_{1}^{1}, \quad \frac{\partial w_{k}}{\partial x_{2}}=-a_{2}^{1} \quad \text { on } \gamma_{i}^{\delta+}, i=1,2, \\
& \left.w_{1 k}\right|_{\gamma_{i}^{\delta_{k^{+}}}}=c_{1}^{1}=c_{1}^{2}, \quad i=1,2,
\end{aligned}
$$

while a construction of the second components $w_{2 k}$ of $W_{k}$ needs more detailed reasonings. We start with constructing auxiliary functions $\mu^{1}(x), \mu^{2}(x), \mu_{k}^{1}(x), \mu_{k}^{2}(x), k=1,2, \ldots$, defined by the relations (see Fig. 2):

$$
\begin{aligned}
& \mu^{1}(x)=-b^{1} x_{1}+c_{2}^{1}, \quad x=\left(x_{1}, 0\right) \in \bar{\gamma}_{1}^{\delta}, \\
& \mu^{2}(x)=-b^{2} x_{1}+c_{2}^{2}, \quad x=\left(x_{1}, 0\right) \in \bar{\gamma}_{2}^{\delta}, \\
& \mu_{k}^{1}(x)=-b^{1} x_{1}+c_{2}^{1}-b^{1}\left(\delta-\delta_{k}\right)+b^{2}\left(\delta-\delta_{k}\right), \quad x=\left(x_{1}, 0\right) \in \bar{\gamma}_{1}^{\delta_{k}}, \\
& \mu_{k}^{2}(x)=-b^{2} x_{1}+c_{2}^{2}, \quad x=\left(x_{1}, 0\right) \in \bar{\gamma}_{2}^{\delta_{k}} .
\end{aligned}
$$

For functions $\tilde{\mu}, \tilde{\mu}_{k}$ defined on $\gamma$ by relations

$$
\tilde{\mu}(x)=\left\{\begin{array}{ll}
\mu^{1}, & x \in \bar{\gamma}_{1}^{\delta}, \\
\mu^{2}, & x \in \bar{\gamma}_{2}^{\delta} ;
\end{array} \quad \tilde{\mu}_{k}(x)= \begin{cases}\mu_{k}^{1}, & x \in \bar{\gamma}_{1}^{\delta_{k}}, \\
\mu_{k}^{2}, & x \in \bar{\gamma}_{2}^{\delta_{k}}\end{cases}\right.
$$

the difference $\theta_{k}(x)=\tilde{\mu}_{k}-\tilde{\mu}$ is well defined for all $x \in \gamma$. We write out the following representation for this function:

$$
\theta_{k}(x)=\theta_{k}\left(x_{1}, 0\right)= \begin{cases}\left(-b^{2} \delta_{k}+c_{2}^{2}\right)-\left(-b^{1} \delta_{k}+c_{2}^{1}\right), & x_{1} \in\left(-1, \delta_{k}\right), \\ \left(-b^{2} x_{1}+c_{2}^{2}\right)-\left(-b^{1} x_{1}+c_{2}^{1}\right), & x_{1} \in\left[\delta_{k}, \delta\right), \\ 0, & x_{1} \in[\delta, 1),\end{cases}
$$

for $\delta_{k}<\delta$; and

$$
\theta_{k}(x)=\theta_{k}\left(x_{1}, 0\right)= \begin{cases}\left(-b^{2} \delta_{k}+c_{2}^{2}\right)-\left(-b^{1} \delta_{k}+c_{2}^{1}\right), & x_{1} \in(-1, \delta), \\ -b^{1}\left(x_{1}-\delta_{k}\right)+b^{2}\left(x_{1}-\delta_{k}\right), & x_{1} \in\left[\delta, \delta_{k}\right), \\ 0, & x_{1} \in\left[\delta_{k}, 1\right),\end{cases}
$$


for cases when $\delta_{k}>\delta$. Note that in both expressions for $\theta_{k}(x)$, the following constant value can be estimated with the help of (17):

$$
\begin{aligned}
& \left(-b^{2} \delta_{k}+c_{2}^{2}\right)-\left(-b^{1} \delta_{k}+c_{2}^{1}\right) \\
& \quad=\left(-b^{2} \delta+c_{2}^{2}\right)-\left(-b^{1} \delta+c_{2}^{1}\right)+b^{2}\left(\delta-\delta_{k}\right)-b^{1}\left(\delta-\delta_{k}\right) \\
& \quad=b^{2}\left(\delta-\delta_{k}\right)-b^{1}\left(\delta-\delta_{k}\right) \leq\left(\left|b^{1}\right|+\left|b^{2}\right|\right)\left|\delta-\delta_{k}\right|, \quad k \in \mathbb{N} .
\end{aligned}
$$

It is evident that $\theta_{k}(x) \in H^{1}(\gamma)$ in both cases when $\delta_{k}<\delta$ and $\delta_{k}>\delta$. Since $\theta_{k}(x)$ is a piecewise linear function, taking into account (19), we can easily obtain the following estimate:

$$
\left\|\theta_{k}(x)\right\|_{H^{1}(\gamma)} \leq C\left|\delta-\delta_{k}\right|
$$

where $C>0$ does not depend on $k \in \mathbb{N}$.

Using the above construction, the following functions are well defined in the corresponding Sobolev spaces:

$$
\begin{aligned}
& \left.w_{2}\right|_{\gamma^{+}}+\theta_{k}(x) \in H^{1 / 2}(\gamma),\left.\quad w_{2}\right|_{\gamma^{-}}+\theta_{k}(x) \in H^{1 / 2}(\gamma), \\
& \left.w_{2}\right|_{\gamma^{+}}+\theta_{k}(x)-\left(\left.w_{2}\right|_{\gamma^{-}}+\theta_{k}(x)\right)=\left[w_{2}\right] \in H_{00}^{1 / 2}(\gamma),\left.\quad w_{2}\right|_{\Gamma} \in H^{1 / 2}(\Gamma)
\end{aligned}
$$

for all $k \in \mathbb{N}$. Applying the linear continuous lifting operator (the corresponding result for lifting operators in the framework of domains with cuts can be found in [1], Lemma 1.12)

$$
\mathcal{L}: H^{1 / 2}(\gamma) \times H^{1 / 2}(\gamma) \times H_{00}^{1 / 2}(\gamma) \times H^{1 / 2}(\Gamma) \rightarrow H^{1}\left(\Omega_{\gamma}\right)
$$

we can construct functions $\tilde{w}_{2}, \tilde{w}_{2 k} \in H^{1}\left(\Omega_{\gamma}\right), k=1,2, \ldots$, with the properties

$$
\begin{aligned}
& \left.\tilde{w}_{2 k}\right|_{\gamma^{+}}=\left.w_{2}\right|_{\gamma^{+}}+\theta_{k}(x),\left.\quad \tilde{w}_{2 k}\right|_{\gamma^{-}}=\left.w_{2}\right|_{\gamma^{-}}+\theta_{k}(x),\left.\quad \tilde{w}_{2 k}\right|_{\Gamma}=\left.\hat{w}_{2}\right|_{\Gamma}, \\
& \left.\tilde{w}_{2}\right|_{\gamma^{+}}=\left.w_{2}\right|_{\gamma^{+}},\left.\quad \tilde{w}_{2}\right|_{\gamma^{-}}=\left.w_{2}\right|_{\gamma^{-}},\left.\quad \tilde{w}_{2}\right|_{\Gamma}=\left.w_{2}\right|_{\Gamma} .
\end{aligned}
$$

Due to continuity of the lifting operator and the uniform estimate (20), we have

$$
\left\|\tilde{w}_{2 k}-\tilde{w}_{2}\right\|_{H^{1}\left(\Omega_{\gamma}\right)} \rightarrow 0 \quad \text { as } k \rightarrow \infty
$$

Then, for the functions $w_{2 k}=\tilde{w}_{2 k}-\tilde{w}_{2}+w_{2}$, it follows

$$
\left\|w_{2 k}-w_{2}\right\|_{H^{1}\left(\Omega_{\gamma}\right)}=\left\|\tilde{w}_{2 k}-\tilde{w}_{2}+w_{2}-w_{2}\right\|_{H^{1}\left(\Omega_{\gamma}\right)} \rightarrow 0 \quad \text { as } k \rightarrow \infty
$$

By construction, we have the equalities

$$
\begin{aligned}
& w_{1 k} \equiv w_{1}, \quad\left[w_{2 k}\right]=\left[w_{2}\right] \quad \text { on } \gamma, \quad w_{2 k}=\mu_{k}^{1}(x) \quad \text { on } \gamma_{1}^{\delta_{k}}, \\
& w_{2 k}=\mu_{k}^{2}(x) \quad \text { on } \gamma_{2}^{\delta_{k}}
\end{aligned}
$$

which guarantee the inclusion $\chi_{k} \in K^{\delta_{k}}, k \in \mathbb{N}$. Relations (21) and (18) provide the convergence

$$
\chi_{k} \rightarrow \chi \quad \text { strongly in } H\left(\Omega_{\gamma}\right) \text {, as } k \rightarrow \infty \text {. }
$$


Keeping in mind the convergences (14), (22), we can pass to the limit as $k \rightarrow \infty$ in inequalities

$$
B\left(\Omega_{\gamma}, \xi^{\delta_{k}}, \chi_{k}-\xi^{\delta_{k}}\right) \geq \int_{\Omega_{\gamma}} F\left(\chi_{k}-\xi^{\delta_{k}}\right) d x
$$

which gives the limit

$$
B\left(\Omega_{\gamma}, \tilde{\xi}, \chi-\tilde{\xi}\right) \geq \int_{\Omega_{\gamma}} F(\chi-\tilde{\xi}) d x
$$

Therefore, by the arbitrariness of $\chi \in K^{\delta}$, we reveal that $\tilde{\xi}=\xi^{\delta}$. Due to the continuous dependence of solutions $\xi^{\delta}$ on $\delta$, we can easily conclude that the function $h(\delta)$ attains minimum and maximum values on the set $[-1 / 2,1 / 2]$ :

$$
p_{1}=\min _{\delta \in[-1 / 2,1 / 2]} h(\delta), \quad p_{2}=\max _{\delta \in[-1 / 2,1 / 2]} h(\delta) .
$$

Finally, as a consequence of the intermediate value theorem for continuous functions, any $p \in\left[p_{1}, p_{2}\right]$ can be fitted within the inverse problem (11), (12). The theorem is proved.

Remark 4 As can be seen from the previous reasonings, the segment $\left[-\frac{1}{2}, \frac{1}{2}\right]$ can be replaced with an arbitrary closed segment lying in $(-1,1)$.

\section{Limit procedure}

In this section we show that a problem for an elastic plate with two bulk hinged inclusions can be obtained by a limit passage from a family of problems for elastic plates with two flat hinged inclusions. In addition to previous assumptions, we suppose that Lipschitz simply connected subdomains $\omega_{1}^{\delta}$ and $\omega_{2}^{\delta}$ have the following properties:

a) $\bar{\omega}_{1}^{\delta} \subset \Omega, \bar{\omega}_{2}^{\delta} \subset \Omega, \bar{\omega}_{1}^{\delta} \cap \bar{\omega}_{1}^{\delta}=(\delta, 0)$;

b) $\gamma_{1}^{\delta} \subset \partial \omega_{1}^{\delta}, \gamma_{2}^{\delta} \subset \partial \omega_{2}^{\delta}$.

We suppose that the given elasticity tensors additionally depend on $\lambda \in(0, \Lambda)$ (where $\Lambda$ is a constant, $0<\Lambda<+\infty)$, as follows:

$$
a_{i j k l}^{\lambda}=\left\{\begin{array}{ll}
a_{i j k l} & \text { in } \Omega \backslash \overline{\omega_{1}^{\delta} \cup \omega_{2}^{\delta}}, \\
\lambda^{-1} a_{i j k l} & \text { in } \omega_{1}^{\delta} \cup \omega_{2}^{\delta},
\end{array} \quad d_{i j k l}^{\lambda}= \begin{cases}d_{i j k l} & \text { in } \Omega \backslash \overline{\omega_{1}^{\delta} \cup \omega_{2}^{\delta}}, \\
\lambda^{-1} d_{i j k l} & \text { in } \omega_{1}^{\delta} \cup \omega_{2}^{\delta} .\end{cases}\right.
$$

Let us define functions

$$
\sigma_{i j}^{\lambda}(W)=a_{i j k l}^{\lambda} \varepsilon_{k l}(W), \quad m_{i j}^{\lambda}(w)=d_{i j k l}^{\lambda} w, k l, \quad i, j=1,2,
$$

and a bilinear form

$$
B^{\lambda}(S, \chi, \bar{\chi})=\int_{S}\left\{\sigma_{i j}^{\lambda}(W) \varepsilon_{i j}(\bar{W})+m_{i j}^{\lambda}(w) \bar{w}, i j\right\} d x
$$

corresponding to some measurable set $S \subset \Omega$. For each fixed $\lambda \in(0, \Lambda)$, we formulate a corresponding variational problem

$$
\Pi^{\lambda}\left(\xi^{\delta, \lambda}\right)=\inf _{\eta \in K^{\delta}} \Pi^{\lambda}(\eta)
$$


where

$$
\Pi^{\lambda}(\chi)=\frac{1}{2} B^{\lambda}\left(\Omega_{\gamma}, \chi, \chi\right)-\int_{\Omega_{\gamma}} F \chi d x, \quad \chi=(W, w) .
$$

According to the line of reasoning in [14], it can be shown that problem (23) has a unique solution and is equivalent to the following variational inequality:

$$
\xi^{\delta, \lambda} \in K^{\delta}, \quad B^{\lambda}\left(\Omega_{\gamma}, \xi^{\delta, \lambda}, \chi-\xi^{\delta, \lambda}\right) \geq \int_{\Omega_{\gamma}} F\left(\chi-\xi^{\delta, \lambda}\right) d x \quad \forall \chi \in K^{\delta} .
$$

Now we justify a limit passage in (24) as $\lambda$ tends to zero. Comparing two inequalities that correspond to (24) with test functions $\chi=2 \xi^{\delta, \lambda}, \chi=0$, we get

$$
B^{\lambda}\left(\Omega_{\gamma}, \xi^{\delta, \lambda}, \xi^{\delta, \lambda}\right)=\int_{\Omega_{\gamma}} F \xi^{\delta, \lambda} d x
$$

Hence, applying inequalities (1), we obtain uniform estimates

$$
\begin{aligned}
& \left\|\xi^{\delta, \lambda}\right\| \leq c, \\
& \frac{1}{\lambda} B\left(\omega_{i}^{\delta}, \xi^{\delta, \lambda}, \xi^{\delta, \lambda}\right) \leq c, \quad i=1,2,
\end{aligned}
$$

where $c>0$ does not depend on $\lambda \in(0, \Lambda)$. Choosing, if necessary, a subsequence, we can assume without loss of generality that there exists a weakly converging in $H\left(\Omega_{\gamma}\right)$ sequence $\left\{\xi^{\delta, \lambda_{n}}\right\}, \xi^{\delta, \lambda_{n}} \rightarrow \tilde{\xi}, \tilde{\xi}=(\tilde{U}, \tilde{u})$ as $\lambda_{n} \rightarrow 0$. In view of (25) we have

$$
\varepsilon_{i j}(\tilde{U})=0, \quad \tilde{u}, i j=0 \quad \text { in } \omega_{k}^{\delta}, \quad i, j, k=1,2 .
$$

The last relations imply that

$$
\tilde{U}=\left(b^{i} x_{2}+c_{1}^{i},-b^{i} x_{1}+c_{2}^{i}\right), \quad \tilde{u}=a_{0}^{i}+a_{1}^{i} x_{1}+a_{2}^{i} x_{2}, \quad x \in \omega_{i}^{\delta},
$$

where $b^{i}, c_{1}^{i}, c_{2}^{i}, a_{0}^{i}, a_{1}^{i}, a_{2}^{i} \in \mathbb{R}, i=1,2$. As it was shown in [14], the function $\tilde{\xi}$ belongs to the set

$$
K_{\omega}^{\delta}=\left\{\chi \in H\left(\Omega_{\gamma}\right) \mid \chi \text { satisfies }(2),\left.\chi\right|_{\omega_{i}^{\delta}}=\zeta^{i}, \zeta^{i} \in R\left(\bar{\omega}_{i}^{\delta}\right), i=1,2\right\} .
$$

Here, we should note that due to properties of trace operators on $H\left(\Omega_{\gamma}\right)$ the conditions of a hinged joint are fulfilled at the point $x^{\delta}$

$$
\begin{aligned}
& \zeta^{1}\left(x^{\delta}\right)=\zeta^{2}\left(x^{\delta}\right), \quad \text { where }\left.\chi\right|_{\omega_{i}^{\delta}}=\zeta^{i}, \zeta^{i} \in R\left(\bar{\omega}_{i}^{\delta}\right), i=1,2, \\
& \frac{\partial w}{\partial x_{1}}=-a_{1}^{1}=-a_{1}^{2}, \quad \frac{\partial w}{\partial x_{2}}=-a_{2}^{1}=-a_{2}^{2},
\end{aligned}
$$

where $\chi=(W, w), \zeta^{i} \in R\left(\bar{\omega}_{i}^{\delta}\right), i=1,2$. Now, performing a passage to the limit in (24) when $\lambda \rightarrow 0$ for an arbitrary test function $\chi \in K_{\omega}^{\delta}$, we obtain

$$
\tilde{\xi} \in K_{\omega}^{\delta}, \quad B\left(\Omega_{\gamma}, \tilde{\xi}, \chi-\tilde{\xi}\right) \geq \int_{\Omega_{\gamma}} F(\chi-\tilde{\xi}) d x \quad \forall \chi \in K_{\omega}^{\delta} .
$$


The last variational inequality is equivalent to the following minimization problem:

$$
\Pi\left(\xi_{\omega}\right)=\inf _{\eta \in K_{\omega}^{\delta}} \Pi(\eta)
$$

which describes an equilibrium of a Kirchhoff-Love plate with two hinged bulk inclusions.

\section{Conclusion}

We have considered the inverse problem (11), (12), which is motivated by applications to fracture mechanics. In this problem it is required to find the location of a hinge point fitting the cost function $J$ in the optimal way. The solvability of the inverse problem is proved rigorously. We have shown that the problem for the plate with two bulk rigid inclusions (26) is connected with the family of problems (23) for plates with flat inclusions in the sense of the limit passage $\lambda \rightarrow 0$. It should be noted that an analogous inverse problem for bulk inclusions seems to be more complicated, and the author has no idea how to prove a similar result to that established in Sect. 3 even for the simplest cases of bulk inclusions, for example, for circular or rhombus rigid inclusions connected in a hinged manner. The case of curvilinear flat inclusions represents another inverse problem subjected for further investigations.

\section{Funding}

The research reported in the second section was supported by the mega-grant of the Russian Federation Government (project no. 14.Y26.31.0013). The research reported in the third section was supported by the Ministry of Science and Higher Education of the Russian Federation within the framework of the base part of the state task FSRG-2020-0006.

\section{Abbreviations}

Not applicable.

Availability of data and materials

Data sharing not applicable to this article as no datasets were generated or analyzed during the current study.

\section{Declarations}

Ethics approval and consent to participate

Not applicable.

Competing interests

The author declares that he has no competing interests.

Authors' contributions

The author read and approved the final manuscript.

\section{Publisher's Note}

Springer Nature remains neutral with regard to jurisdictional claims in published maps and institutional affiliations.

Received: 14 June 2021 Accepted: 9 October 2021 Published online: 04 November 2021

References

1. Khludnev, A.M., Kovtunenko, V.A.: Analysis of Cracks in Solids. WIT Press, Southampton (2000)

2. Faella, L., Khludnev, A.: Junction problem for elastic and rigid inclusions in elastic bodies. Math. Methods Appl. Sci. 39(12), 3381-3390 (2016)

3. Khludnev, A.M., Faella, L., Popova, T.S.: Junction problem for rigid and Timoshenko elastic inclusions in elastic bodies. Math. Mech. Solids 22(4), 737-750 (2017)

4. Shcherbakov, V.V.: The Griffith formula and J-integral for elastic bodies with Timoshenko inclusions. Z. Angew. Math. Mech. 96, 1306-1317 (2016)

5. Shcherbakov, V.V.: Shape optimization of rigid inclusions for elastic plates with cracks. Z. Angew. Math. Phys. 67(3), 71 (2016) 
6. Knees, D., Schroder, A.: Global spatial regularity for elasticity models with cracks, contact and other nonsmooth constraints. Math. Methods Appl. Sci. 35(15), 1859-1884 (2012)

7. Lazarev, N.P., Semenova, G.M.: Equilibrium problem for a Timoshenko plate with a geometrically nonlinear condition of nonpenetration for a vertical crack. J. Appl. Ind. Math. 14(3), 532-540 (2020)

8. Lazarev, N.P.: Equilibrium problem for an thermoelastic Kirchhoff-Love plate with a nonpenetration condition for known configurations of crack edges. Siberian Electron. Math. Rep. 17, 2096-2104 (2020)

9. Itou, H., Kovtunenko, V.A., Rajagopal, K.R.: Nonlinear elasticity with limiting small strain for cracks subject to non-penetration. Math. Mech. Solids 22(6), 1334-1346 (2017)

10. Lazarev, N.P., Rudoy, E.M.: Optimal size of a rigid thin stiffener reinforcing an elastic plate on the outer edge. Z. Angew. Math. Mech. 97(9), 1120-1127 (2017)

11. Lazarev, N.P., Semenova, G.M., Romanova, N.A.: On a limiting passage as the thickness of a rigid inclusions in an equilibrium problem for a Kirchhoff-Love plate with a crack. J. Sib. Fed. Univ. Math. Phys. 14(1), $28-41$ (2021)

12. Cai, D.-L., Sofonea, M., Xiao, Y.-B.: Convergence results for elliptic variational-hemivariational inequalities. Adv. Nonlinear Anal. 10(1), 2-23 (2021)

13. Zeng, S., Bai, Y., Gasinski, L., Winkert, P.: Convergence analysis for double phase obstacle problems with multivalued convection term. Adv. Nonlinear Anal. 10(1), 659-672 (2021)

14. Khludnev, A.M.: Problem of a crack on the boundary of a rigid inclusion in an elastic plate. Mech. Solids 45(5), 733-742 (2010)

15. Khludnev, A.M.: On bending an elastic plate with a delaminated thin rigid inclusion. J. Appl. Ind. Math. 5(4), 582-594 (2011)

16. Khludnev, A.: Thin rigid inclusions with delaminations in elastic plates. Eur. J. Mech. A, Solids 32, 69-75 (2012)

17. Lazarev, N., Itou, H.: Optimal location of a rigid inclusion in equilibrium problems for inhomogeneous Kirchhoff-Love plates with a crack. Math. Mech. Solids 24(12), 3743-3752 (2019)

18. Lazarev, N., Everstov, V:: Optimal location of a rigid inclusion in equilibrium problems for inhomogeneous two-dimensional bodies with a crack. Z. Angew. Math. Mech. 99(3), e201800268 (2019)

19. Rudoy, E.M.: Shape derivative of the energy functional in a problem for a thin rigid inclusion in an elastic body. Z. Angew. Math. Phys. 66(4), 1923-1937 (2015)

20. Neustroeva, N.V.: A rigid inclusion in the contact problem for elastic plates. J. Appl. Ind. Math. 4(4), 526-538 (2010)

21. Namm, R.V., Tsoy, G.I.: Solution of a contact elasticity problem with a rigid inclusion. Comput. Math. Math. Phys. 59, 659-666 (2019)

22. Rudoy, E.M.: Numerical solution of an equilibrium problem for an elastic body with a thin delaminated rigid inclusion. J. Appl. Ind. Math. 10, 264-276 (2016)

23. Lazarev, N.: Existence of an optimal size of a delaminated rigid inclusion embedded in the Kirchhoff-Love plate. Bound. Value Probl. 2015, 180 (2015)

24. Rudoy, E., Shcherbakov, V.: First-order shape derivative of the energy for elastic plates with rigid inclusions and interfacial cracks. Appl. Math. Optim. (2020). https://doi.org/10.1007/s00245-020-09729-5

25. Khludnev, A., Esposito, A.C., Faella, L.: Optimal control of parameters for elastic body with thin inclusions. J. Optim. Theory Appl. 184(1), 293-314 (2020)

26. Khludnev, A.M., Popova, T.S.: On junction problem with damage parameter for Timoshenko and rigid inclusions inside elastic body. Z. Angew. Math. Mech. 100(8), e202000063 (2020)

27. Khludnev, A.M.: Inverse problems for elastic body with closely located thin inclusions. Z. Angew. Math. Phys. 70(5), 134 (2019)

28. Khludnev, A.M., Shcherbakov, V.V.: Singular path-independent energy integrals for elastic bodies with Euler-Bernoulli inclusions. Math. Mech. Solids 22(11), 2180-2195 (2017)

29. Sokołowski, J. Żochowski, A.: Topological derivatives for optimization of plane elasticity contact problems. Eng. Anal. Bound. Elem. 32, 900-908 (2008)

30. Sokołowski, J., Zolésio, J.P.: Introduction to Shape Optimization. Shape Sensitivity Analysis. Springer Ser. Comput. Math., vol. 16. Springer, Berlin (1992)

31. Ghilli, D., Kunisch, K., Kovtunenko, V.A.: Inverse problem of breaking line identification by shape optimization J. Inverse III-Posed Probl. 28(1), 119-135 (2020)

32. Cakoni, F., Kovtunenko, V.A.: Topological optimality condition for the identification of the center of an inhomogeneity. Inverse Probl. 34(3), 035009 (2018)

33. Novotny, A.A., Sokołowski, J.: Topological Derivatives in Shape Optimization. Series: Interaction of Mechanics and Mathematics. Springer, Berlin (2013)

34. Hintermüller, M., Laurain, A.: Optimal shape design subject to variational inequalities. SIAM J. Control Optim. 49 , 1015-1047 (2011)

35. Furtsev, A., Itou, H., Rudoy, E.: Modeling of bonded elastic structures by a variational method: theoretical analysis and numerical simulation. Int. J. Solids Struct. 182-183, 100-111 (2020)

36. Leugering, G., Sokolowski, J., Zochowski, A.: Control of crack propagation by shape-topological optimization. Discrete Contin. Dyn. Syst., Ser. A 35(6), 2625-2657 (2015)

37. Kovtunenko, V.A., Leugering, G.: A shape-topological control problem for nonlinear crack-defect interaction: the antiplane variational model. SIAM J. Control Optim. 54(3), 1329-1351 (2016)

38. Bilotta, A., Morassi, A., Rosset, E., Turco, E., Vessella, S.: Numerical size estimates of inclusions in Kirchhoff-Love elastic plates. Int. J. Solids Struct. 168, 58-72 (2019)

39. Morassi, A., Rosset, E., Vessella, S.: Stable determination of a rigid inclusion in an anisotropic elastic plate. SIAM J. Math Anal. 44, 2204-2235 (2012)

40. Morassi, A., Rosset, E., Vessella, S.: Optimal stability in the identification of a rigid inclusion in an isotropic Kirchhoff-Love plate. SIAM J. Math. Anal. 51, 731-747 (2019) 